\title{
Fabrication of Non-Ionic Surfactant Vesicular Gel for Effective Treatment of Rheumatoid Arthritis
}

\author{
Subashini Rajaram ${ }^{1}$, Anusuriya S. ${ }^{2}$, Senthil Rajan Dharmalingam ${ }^{3}$, Kumarappan Chidambaram ${ }^{4}$
}

${ }^{1}$ Department of Pharmaceutics and Research, Swamy Vivekanandha College of Pharmacy, Tiruchengode, Tamilnadu, India. '2Department of Pharmaceutics and Research, Swamy Vivekanandha College of Pharmacy,

Tiruchengode, Tamilnadu, India. ${ }^{3}$ Department of Pharmaceutics and Research, Swamy Vivekanandha College of Pharmacy, Tiruchengode, Tamilnadu, India. ${ }^{4}$ Department of Pharmacology and Toxicology, School of Pharmacy, King Khalid University, Saudi Arabia.

\section{ABSTRACT}

\section{BACKGROUND}

Rheumatoid Arthritis (RA) is a chronic, inflammatory auto immune disorder with unknown aetiology. Though various efficacious treatment options are currently available to treat RA, failure to cure is unpredictable. Hence the present study aimed to evaluate Piroxicam (PC) loaded non-ionic surfactant vesicular carrier as transdermal patches.

\section{METHODS}

Transdermal patches of PC- niosomes were formulated by solvent casting method using different polymers. The prepared formulation was examined for physico chemical and morphological characteristics and drug release studies were performed by Franz diffusion cell method.

\section{RESULTS}

The results of physico-chemical characterizations show that all formulations were with optimum range and morphological characterization shows that the prepared niosomes are spherical in shape. Drug release characteristic of all formulations was performed and the result exhibits that formulation TN4 (PC encapsulated niosomal gel transdermal patch) shows highest \% drug release (95.13\%) when compared to other formulation. The release data was fitted with release kinetics and results shows zero order with non Fickian diffusion mechanism.

\section{CONCLUSIONS}

PC encapsulated vesicular carrier as transdermal patches is a promising drug delivery system to enhance the solubility of poorly soluble drugs. It also enhances the residence time of drug at absorption site. Hence this approach could be beneficial for the effective management of RA.

\section{KEY WORDS}

Niosomes, Rheumatoid Arthritis, Piroxicam, and Transdermal Drug Delivery System
Corresponding Author: Dr. Subashini Rajaram, Associate Professor, Swamy Vivekanandha College of Pharmacy,

Tiruchengode, Namakkal-637201,

Tamilnadu, India.

E-mail: subababu.r@gmail.com

DOI: $10.14260 /$ jemds/2020/496

How to Cite This Article:

Rajaram $S$, Anusuriya $S$, Dharmalingam $S R$, et al. Fabrication of non-ionic surfactant vesicular gel for effective treatment of rheumatoid arthritis. J. Evolution Med. Dent. Sci. 2020;9(32): 2289-2295, DOI:

10.14260/jemds/2020/496

Submission 02-04-2020,

Peer Review 28-06-2020,

Acceptance 06-07-2020,

Published 10-08-2020.

Copyright (C) 2020 JEMDS. This is an open access article distributed under Creative Commons Attribution License [Attribution 4.0 International (CC BY 4.0)] 


\section{BACKGROUND}

Rheumatoid arthritis (RA) is an autoimmune, chronic inflammatory disorder, affecting the joints. It causes a painful swelling that leads to bone erosion and joint deformity. It occurs when the immune system mistakenly attacks the synovium (the lining of the membranes surrounding the joints). It causes inflammation of the tissue lines within the joints, results in swelling and pain in and around the joints. Finally, it destroys the cartilage and bone within the joints. RA affects the joints of the hands, feet, wrists, elbows, knees and ankles. The joint effect is usually symmetrical. The entire body system is affected by RA. 1

In general, increasing level of prostaglandin leads to produce pain in our body. NSAID (Non-Steroidal AntiInflammatory Drugs) are therapeutic agents used for the management of pain. It can be minimized by the drug Piroxicam (PC) and it also coming under the category of NSAID. Literature reveals that most of the inflammatory conditions such as rheumatoid arthritis, osteoarthritis, postoperative pain, musculoskeletal disorders, and dysmenorrhoeal were treated by PC. It also minimizes the levels of prostaglandins by blocking the enzyme that produces prostaglandins (cyclooxygenase), and other chemicals that are responsible for pain, fever, and inflammation. ${ }^{2}$

PC is a Class II drug with low solubility and high permeability. It is well absorbed through oral administration and also has long elimination half-life. Its use has been limited due to its side effects such as bleeding, abdominal pain, gastritis, drowsiness, nausea, vomiting, dizziness, pruritis and ulceration. Hence an alternative drug delivery system to be needed to overcome the lacuna with the side effects associated with the oral route of PC. ${ }^{3}$

Transdermal drug delivery system (TDDS) is an attractive alternative drug delivery system for from various novel drug delivery systems. It has been developed to enhance the systemic availability of drugs through topical application with predetermined rate. It also shows minimal inter and intra patient variation. In transdermal drug delivery system skin acts as a principle barrier in drug delivery. ${ }^{3}$ Italso overcome gastrointestinal problems associated with drugs and low absorption. ${ }^{4}$ The effective concentration of drug for an extended period in systemic circulation can be achieved through bypass hepatic first pass metabolism by the formulation of transdermal patches of PC with the utilization of niosome as vesicular carriers.

In our study we selected niosome as vesicular carriers due to its stability and economic over others. Previous study reveals that niosomes consists of various concentric bilayer membrane mainly composed of nonionic surfactants and cholesterol enclosing aqueous phase in the core. It enhances the solubility of poorly soluble drug by extended drug action thus improves its bioavailability also. Enhancement of penetration through biological membrane can be achieved by surfactants through biological membrane interaction, adsorption at interfaces and modification in the barrier function of subcutaneous tissue. It results reversible modification of lipids. ${ }^{5}$ Thus the present study aimed to fabricate PC encapsulated niosomal gel and its characterization for its functionality as a transdermal carrier in delivering therapeutically effective amount of PC through in-vitro release study against RA.

\section{METHODS}

Piroxicam was gifted from Heer Pharm Pvt Ltd, Mumbai, India, Cholesterol was purchased from Dishman Carbogen Amcis Ltd., Delhi, Carbopol was purchased from Maruti Chem. Industries, Gujarat, Chloroform was purchased from Avi Chem. industries, Mumbai, Methanol and Ethanol were purchased from Karin Scientific Products, Chennai, Propylene glycol was purchased from India Glycols, Chennai, Triethanolamine was purchased from Ultimate Chem. Industries Pvt Ltd., Mumbai, and Tween 80 was purchased from Continental Chemicals Pvt Ltd., Delhi. All the chemicals used were of analytical grade. All solutions were prepared using double distilled water.

\section{Preparation of Piroxicam-Loaded Niosome}

Thin - film hydration method was used to prepare PC encapsulated niosome using various concentrations of cholesterol and tween 80 and the formula is given in Table.1. Tween 80, cholesterol, and $20 \mathrm{mg}$ of PC were dissolved in a mixture of chloroform and methanol (7:3v/v ratio) in a 250 $\mathrm{mL}$ round bottom flask. A thin, dry, uniform film was formed by evaporating the solvent in the rotary flash evaporator. Niosomal dispersion was formed by the hydration of obtained film using phosphate buffered saline ( $\mathrm{pH}$ 7.4). Conversion of multilamellar vesicles to desired size unilamellar vesicles was done by sonication process using probe sonicator and it was centrifuged at $4,000 \mathrm{rpm}$ and $4^{\circ} \mathrm{C}$ for $15 \mathrm{~min}$ by laboratory centrifuge (Remi P-R-24, India) to separate unentrapped drug. Supernatant liquid was decanted and quantified for vesicle size and \% drug entrapment (PDE). The unentrapped drug was measured from the sediment obtained at the bottom of the centrifuge tube. Best formulation was selected based on its maximum drug entrapment efficiency with desirable particle size. ${ }^{5}$

\section{Preparation of Niosomal Gel}

Based on its maximum drug entrapment efficiency with desirable particle size the best formulation (F3c) was selected as best one for further preparation. Niosomal gel was prepared from $10 \mathrm{~mL}$ of niosomal dispersion by adding $200 \mathrm{mg}$ of Carbopol 934 with continuous stirring using a glass rod. Glycerol (10\%) and Triethanolamine (q.s) that made it transparent was added drop wise for neutralization till reaching the desired skin $\mathrm{pH}$ (6.8-7). Plain PC gel was formulated using similar procedure with hydroethanolic solution $(15 \%(\mathrm{v} / \mathrm{v})$ ethanol) of PC. All the samples were stored in refrigerator $\left(4^{\circ} \mathrm{C}\right)$ for at least 24 hours. ${ }^{6}$

\section{Preparation of Niosomal Gel Encapsulated Transdermal Patch}

Transdermal patches of PC- niosomes were formulated by solvent casting method using different polymers like ethyl cellulose (EC), polyvinylpyrrolidone (PVP), Eudragit L100 as 
per the formula given in Table 1. A weighed amount of PVA $(2.5 \% \mathrm{w} / \mathrm{v})$ was added to a required volume of warm distilled water and a homogenous solution was made by constant stirring and intermittent heating at $60^{\circ} \mathrm{C}$ for a few seconds and poured into glass moulds already wrapped with aluminium foil around open ends and were kept for drying at $60^{\circ} \mathrm{C}$ for $6 \mathrm{~h}$, forming a smooth, uniform, and transparent backing membrane. Backing membrane was used as a support for drug-polymer matrix. Then, the niosomal dispersion was added slowly in the polymeric solution and stirred with the help of magnetic stirrer to obtain a uniform solution. $0.1 \%$ of Propylene glycol (PG) was used as a plasticizer. Then the solution was poured on the glass moulds of $5 \mathrm{~cm} \times 5 \mathrm{~cm}$ and dried at the room temperature. The prepared patches were cut into $1 \mathrm{~cm} \times 1 \mathrm{~cm}$ in size and stored at $40^{\circ} \mathrm{C}$ and $75 \%$ relative humidity in polyethylene bag for further process. ${ }^{4}$

\begin{tabular}{|ccccc|}
\hline $\begin{array}{c}\text { Sl. } \\
\text { No. }\end{array}$ & $\begin{array}{c}\text { Formulation } \\
\text { Code }\end{array}$ & $\begin{array}{c}\text { Piroxicam } \\
\text { (mg) }\end{array}$ & $\begin{array}{c}\text { Composition } \\
\text { Cholesterol } \\
\text { (gm) }\end{array}$ & $\begin{array}{c}\text { Tween 80 } \\
\text { (mL) }\end{array}$ \\
1. & F1 & 20 & - & 0.5 \\
2. & F2 & 20 & - & 1 \\
3. & F3 & 20 & - & 1.5 \\
4. & F3a & 20 & 0.2 & 1.0 \\
5. & F3b & 20 & 0.4 & 0.5 \\
6. & F3c & 20 & 0.6 & 1.5 \\
\hline \multicolumn{5}{r}{ Table 1. Formulation of Noisome } \\
\hline
\end{tabular}

\begin{tabular}{|cccccc|}
\hline \multirow{2}{*}{ S1. No. } & \multirow{2}{*}{ Ingredients } & Composition (mg/mL) \\
1. & Niosomal dispersion & 10 & TN2 & TN3 & TN4 \\
2. & PVP & 300 & - & 10 & 10 \\
3. & Eudragit L 100 & 100 & 200 & - & 400 \\
4. & EC & - & 150 & 200 & - \\
\hline \multicolumn{5}{c|}{} \\
\hline
\end{tabular}

TN1- Transdermal patch consists of F3c with PVP and EC.

TN2- Transdermal patch consists of F3c with Eudragit L 100 and EC.

TN3- Transdermal patch consists of F3c with PVP and Eudragit L 100.

TN4- Transdermal patch consists of Niosomal gel with TN3.

\section{Characterization of Niosomes}

Fourier Transform Infrared Spectrometric Method (FT-IR)This study was carried out to examine the interaction between drug and polymers in the formulations. FTIR spectrum of pure drug, physical mixtures and solid dispersions were recorded by using FT-IR $8400 \mathrm{~S}$ (SHIMADZU, Kyoto, Japan) with the scanning range 4000 to $400 \mathrm{~cm}^{-1} .^{7}$

Vesicular Shape and Surface Morphology- Initial visualization of prepared niosomes was done by Olympus BX 40 microscope (at 40X) before sonication and Photomicrographs were taken. Followed by surface morphology of niosomal dispersion was carried by Scanning electron microscope (JSM-5610LV, JEOL, Japan). ${ }^{5}$ Particle Size, Zeta Potential and Polydispersity Index (PDI)
Niosomal dispersions were ten times diluted with distilled water and the diameter of niosomes was determined using a Zetamaster S particle electrophoresis and particle size analyser (Malvern Instruments Ltd., Malvern, UK). The instrument was used to measure both particle size (Photon Correlation Spectroscopy - PCS) and zeta potential. 8 The poly dispersity index (PDI), which is the width of the particle size distribution curve, was determined as a measure of the homogeneity. Small values of PDI $<0.1$ indicate a homogeneous population, while a PDI $>0.3$ indicates its high heterogeneity. ${ }^{8}$

Percentage of Drug Entrapment (PDE)- UV-visible spectrophotometer (UV-1700, Shimadzu) was used to determine the amount of drug entrapped in niosomal dispersion at $335 \mathrm{~nm}$. The amount of drug entrapped, and unentrapped portion of PC were dissolved separately in a mixture of methanol (7:3 ratio) and the difference in absorbance $(\mathrm{dA} / \mathrm{d} \lambda)$ were observed at $\lambda \max$ of $335 \mathrm{~nm}$. The PDE was finally calculated using the formula: ${ }^{5}$

Percentage Drug Entrapment =

\section{(Amount of Entraped Drug x 100) Total Amount of Drug}

\section{Characterization of Niosomal Gel}

The niosomal gel and plain topical PC gel were characterized with respect to its Physical appearance, $\mathrm{pH}$, and viscosity.

Physical Appearance- Clarity, Colour and the presence of foreign particles in the niosomal gel was determined. ${ }^{9}$

$\mathrm{pH}$ Measurements- Digital $\mathrm{pH}$ meter was used to identify the $\mathrm{pH}$ of the gel formulations. $\mathrm{pH}$ meter was calibrated before determination of $\mathrm{pH}$ and the readings were taken by dipping the glass rod into the gel formulations.

Viscosity Measurement- Brookfield (DV-E) viscometer was used to determine the viscosity of gel formulations. $25.0 \mathrm{~g}$ gel was taken in beaker and spindle number 4 was rotated at $50 \mathrm{rpm}$ and viscosity was recorded. ${ }^{9}$

\section{Characterization of Transdermal Patch}

Thickness- The thickness of transdermal film is determined by a dial callipers [Aeroscape $(150 \times 0.2 \mathrm{~mm}$ ] at different points of the patch and the average values were calculated. 10

Uniformity of Weight- Weight uniformity test was done in three individual patches and average weight was calculated. 10 
Moisture Content- The moisture content of the prepared films was analysed by the method described by Dey SK et al., 2011. The percent moisture content is calculated using following formula. 10

Percentage moisture content $=$

$$
\frac{\text { Initial weight-Final weight }}{\text { Final weight }} \times 100
$$

Folding Endurance- Folding endurance gives the withstanding capacity of films while subjected to frequent extreme folding conditions. It is determined by folding the film repeatedly at the same point until it breaks, and folding endurance value is the number of times the films could be folded at the same place without breaking. This indicates the brittleness of films. ${ }^{10}$

\section{In-Vitro Release Studies on Topical Gel}

The In-vitro studies of plain topical gel and niosomal gel were carried out with Franz diffusion (FD) cell. The gel was placed in a dialysis membrane with a molecular cut-off of (MWCO $12,000-15,000 \mathrm{Da}$, HiMedia, India) which acts as a donor compartment and placed into $\mathrm{pH} 7.4$ phosphate buffer as media in a beaker which was taken in receptor compartment. The entire system was kept at $37^{\circ} \mathrm{C} \pm 0.1^{\circ} \mathrm{C}$ with continuous magnetic stirring at a rotation speed of $50 \mathrm{rpm}$. At appropriate time intervals $(0,15,30,45,60 \mathrm{~min}, 2,4,6,8,12$, $24 \mathrm{hrs}) 1 \mathrm{~mL}$ of the release medium was removed through $0.1 \mu \mathrm{m}$ membrane filtered immediately and maintained sink condition by the addition of $1 \mathrm{~mL}$ of fresh 7.4 phosphate buffer solution to the system. The amount of PC released in the medium was determined by UV-Vis spectrophotometer at $335 \mathrm{~nm}$. The experiment was run in triplicate and the mean values were recorded as percent release of PC. The results are expressed as a cumulative percentage of the released drug, which calculated by the given equation. ${ }^{9}$

Cumulative drug release $(\%)=$

$$
\frac{\text { Amount of drug released at time } \mathrm{t}(\mathrm{mg})}{\text { Total amount of drug }(\mathrm{mg})} \times 100
$$

\section{In-Vitro Release Studies on Transdermal Patch}

In-vitro release study on niosomal dispersion and niosomes loaded transdermal patches was performed in a Franz diffusion assembly of capacity $10 \mathrm{~mL}$ using dialysis membrane (MWCO 12,000-15,000Da, HiMedia, India) using phosphate buffer $\mathrm{pH}$ 7.4. The temperature of the diffusion cell was maintained at $37 \pm 0.5^{\circ} \mathrm{C}$ by circulating water jacket. Stirring was continued in the receiver compartment using magnetic bead until the process completion. $1 \mathrm{~mL}$ sample was withdrawn at each time intervals and sink condition was maintained by the addition of an equal amount of phosphate buffer $\mathrm{pH}$ 7.4. Absorbance was recorded Spectrophotometrically at $335 \mathrm{~nm}$. The cumulative percentage of drug diffused at each time interval was calculated. The experiment was run in triplicate and the mean values were recorded as percent release of PC. The results are expressed as a cumulative percentage of the released drug, which calculated by the given equation. 11

Cumulative drug release $(\%)=$

$$
\frac{\text { Amount of drug released at time } \mathrm{t}(\mathrm{mg})}{\text { Total amount of drug }(\mathrm{mg})} \times 100
$$

\section{Kinetic Model of In Vitro Drug Release Studies}

The in vitro release studies data were fitted to various kinetic models like Zero order, first order, Higuchi Plot and Koresmeyer-Peppas plot to identify the model of drug release. ${ }^{12}$

\section{Statistical Analysis}

The data were analysed by one-way ANOVA followed by Tukey's multiple comparison tests with the help of Graph Pad InStat software, version 6.01. All the data were presented as a mean value with its standard deviation (mean \pm S.D). $\mathrm{P}<0.05$

\begin{tabular}{|c|c|c|c|c|c|c|c|c|}
\hline $\begin{array}{c}\text { Formulation } \\
\text { Code }\end{array}$ & \multicolumn{2}{|c|}{$\begin{array}{c}\text { Particle } \\
\text { Size }(\mu \mathrm{m})\end{array}$} & \multicolumn{2}{|c|}{$\begin{array}{c}\text { Zeta } \\
\text { Potential } \\
\text { mV) }\end{array}$} & \multicolumn{2}{|c|}{ PDI } & \multicolumn{2}{|c|}{$\begin{array}{c}\text { Entrapment } \\
\text { Efficiency (\%) }\end{array}$} \\
\hline F1 & \multicolumn{2}{|c|}{$595.21 \pm 1.52$} & \multicolumn{2}{|c|}{$32.1 \pm 6.78$} & \multicolumn{2}{|c|}{$0.11 \pm 2.5$} & \multicolumn{2}{|c|}{$53.12 \pm 3.12$} \\
\hline F2 & \multicolumn{2}{|c|}{$591.3 \pm 1.23$} & \multicolumn{2}{|c|}{$38.6 \pm 5.48$} & \multicolumn{2}{|c|}{$0.19 \pm 1.09$} & \multicolumn{2}{|c|}{$64.37 \pm 2.5$} \\
\hline F3 & \multicolumn{2}{|c|}{$529.8 \pm 2.35$} & \multicolumn{2}{|c|}{$39.1 \pm 4.6$} & \multicolumn{2}{|c|}{$0.25 \pm 2.31$} & \multicolumn{2}{|c|}{$68.93 \pm 2.52$} \\
\hline F3a & \multicolumn{2}{|c|}{$631.2 \pm 4.13$} & \multicolumn{2}{|c|}{$41.0 \pm 2.31$} & \multicolumn{2}{|c|}{$0.27 \pm 3.3$} & \multicolumn{2}{|c|}{$83.75 \pm 4.68$} \\
\hline F3b & \multicolumn{2}{|c|}{$642.9 \pm 5.17$} & \multicolumn{2}{|c|}{$45.2 \pm 7.4$} & \multicolumn{2}{|c|}{$0.31 \pm 0.51$} & \multicolumn{2}{|c|}{$90.12 \pm 4.68$} \\
\hline F3c & \multicolumn{2}{|c|}{$728.3 \pm 7.22$} & \multicolumn{2}{|c|}{$46.9 \pm 9.83$} & \multicolumn{2}{|c|}{$0.39 \pm 0.011$} & \multicolumn{2}{|c|}{$97.73 \pm 7.81$} \\
\hline Formulation & \multicolumn{2}{|c|}{ Colour } & \multicolumn{2}{|c|}{ Clarity } & \multicolumn{2}{|c|}{$\mathrm{pH}$} & \multicolumn{2}{|c|}{ Viscosity (cps) } \\
\hline Plain gel & \multicolumn{2}{|c|}{ White } & & Clear & $6.3 \pm 1$ & & & $69 \pm 1.47$ \\
\hline Niosomal gel & Whit & & & Clear & $6.5 \pm 0$ & & & $5 \pm 1.51$ \\
\hline $\begin{array}{c}\text { Formulation } \\
\text { Code }\end{array}$ & $\begin{array}{r}\text { Weig } \\
\text { Unifor } \\
\text { (mg }\end{array}$ & & & $\begin{array}{l}\text { lding } \\
\text { urance }\end{array}$ & $\begin{array}{r}\text { Thickn } \\
\text { (mm }\end{array}$ & & & $\begin{array}{l}\text { Moisture } \\
\text { content }\end{array}$ \\
\hline $\begin{array}{l}\text { Niosomal } \\
\text { dispersion }\end{array}$ & $44.1 \pm$ & & & $0.3 \pm 0.98$ & $0.257 \pm$ & .001 & & $41 \pm 0.04$ \\
\hline TN1 & 53.7 & & & 1.63 & 0.268 & & & 2 \\
\hline TN2 & $54.1 \pm$ & & & $87 \pm 3.3$ & $0.277 \pm$ & 02 & & $21 \pm 0.02$ \\
\hline TN3 & 56.5 & & & $4.6 \pm 2.05$ & 0.285 & & & $89 \pm 0.03$ \\
\hline TN4 & $53.36 \pm$ & & & $1 \pm 1.63$ & $0.295 \pm$ & .002 & & $73 \pm 0.03$ \\
\hline Table & Char & & & esul & fAll Fo & & or & \\
\hline Sl. Formulation & $\begin{array}{l}\text { Zero } \\
\text { Order }\end{array}$ & $\begin{array}{l}\text { Fi } \\
\text { Or }\end{array}$ & & $\begin{array}{r}\text { Kors } \\
\text { Pe| }\end{array}$ & $\begin{array}{l}\text { eyer } \\
\text { las }\end{array}$ & & & Hixs \\
\hline & $\mathbf{R}^{2}$ & & & $\mathbf{R}^{2}$ & n & & & \\
\hline 1. $\begin{array}{c}\text { Niosomal } \\
\text { dispersion }\end{array}$ & 0.886 & 0.8 & & 0.98 & 0.663 & 0.9 & 3 & 0.989 \\
\hline TN1 & 0.879 & 0.8 & & 0.991 & 0.780 & $0 . c$ & 3 & 0.897 \\
\hline $\mathrm{TN} 2$ & 0.858 & 0.8 & & 0.987 & 0.633 & $0 . c^{2}-x-5$ & 985 & 0.873 \\
\hline TN3 & 0.884 & 0.8 & & 0.987 & 0.714 & & 895 & 0.896 \\
\hline TN4 & 0.946 & & & 0.998 & 0.817 & & 981 & 0.962 \\
\hline
\end{tabular}
was considered as statistically significant.

\section{RESULTS}

\section{Scanning Electron Microscopy}

The Particle Shape and surface characteristics of all formulations were examined by Scanning Electron Microscopy and the figure 1 illustrated for the best formulation F3c.

\section{Characterization of Niosomes, Gel, and Transdermal Patches}

The Particle size, zeta potential and poly dispersity index (PDI) of all formulations was determined and mean data was given in Table. 2. 

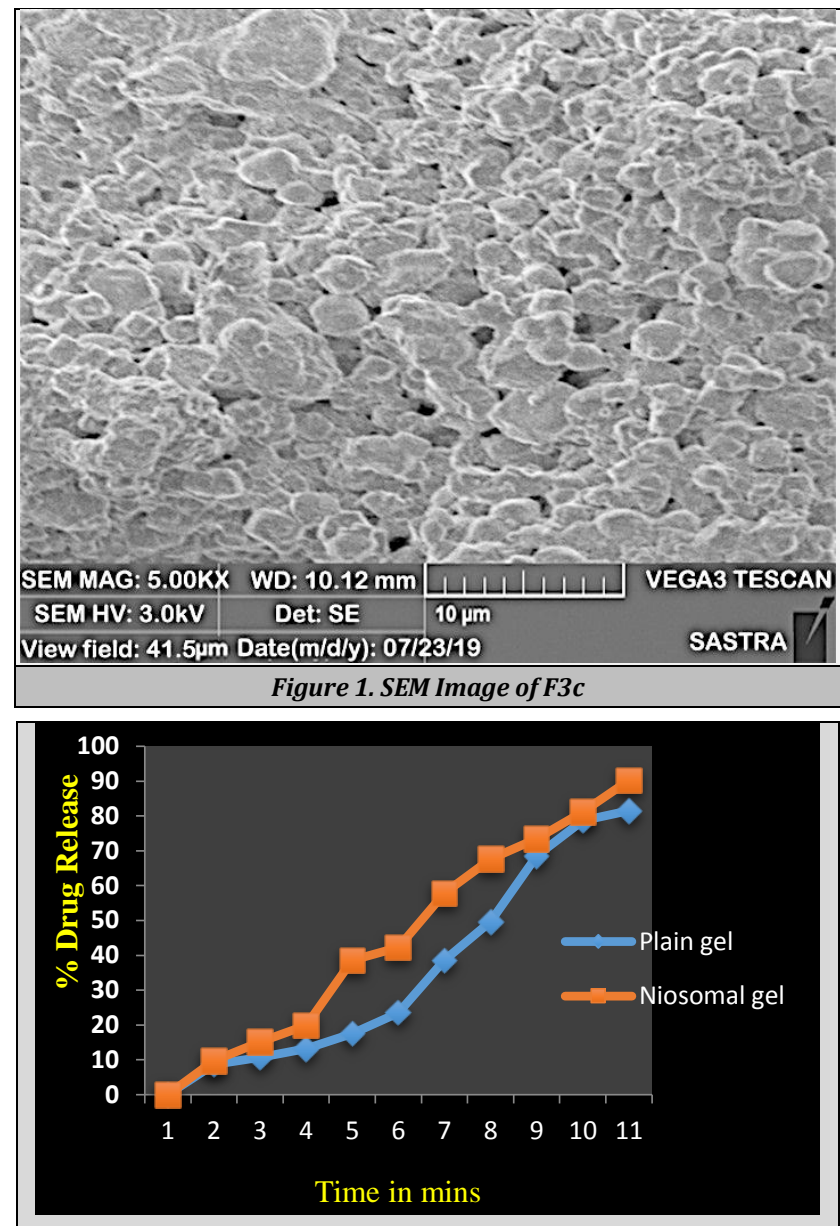

In-Vitro Release Studies on Topical Gel

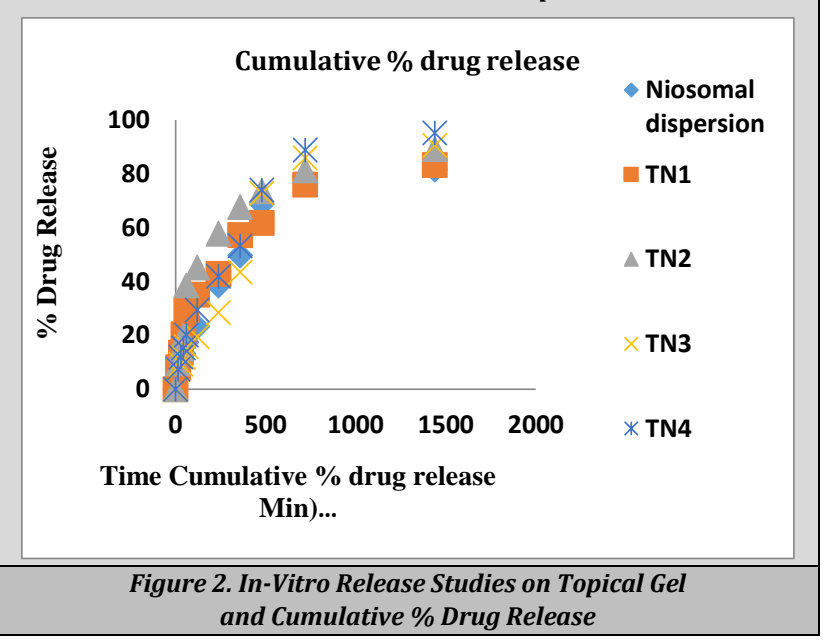

\section{DISCUSSION}

\section{Fourier Transform Infrared Spectrometric Method (FT-IR)}

In compatibility study, IR Spectroscopy can be used to identify a compound and also to investigate the composition of the mixture. IR spectral studies of pure PC, Polymer and their formulations have higher proportion and result shown that drug and polymer was compatible with each other in all preparations.
Particle Size Analysis, Zeta Potential and Polydispersity Index (PDI)

The Particle size, zeta potential and poly dispersity index (PDI) of all formulations was determined and mean data was given in Table.2. It shows that when the concentration of surfactant increases the size of niosomes also increase. This is due to the surface free energy decreases with an increase in hydrophobicity of surfactant. 13

Zeta potential is one of the parameters for interpreting the stability of colloidal systems. As the zeta potential increases, the charged particles repel one another, and this stabilizes the system against aggregation. Systems with the zeta potential value of $\pm 30 \mathrm{mV}$ or higher are considered to be stable ${ }^{[14]}$ due to presence of electrical repulsive forces among the vesicles.[15] Zeta potential of niosomal formulations was found in the range of 32.1 to $46.9 \pm 9.83 \mathrm{mV}$. The result of zeta potential reveals that all the prepared formulations are stable with highest zeta potential value.

The polydispersity index (PDI), which is the width of the particle size distribution curve, was determined as a measure of the homogeneity. Small values of PDI $<0.1$ indicate a homogeneous population, while a PDI $>0.3$ indicates its high heterogeneity. The PDI of all niosomal formulations was found in range between 0.11 to 0.39 which shown that homogenous nature of formulations.

\section{Percentage Entrapment Efficiency (PDE)}

The drug encapsulation efficiency in vesicular system relies on the stability of vesicle in formulation. In general, stability is highly dependent on the type and quantity of surfactant used because it forms bilayer in vesicular system. The mean result of \% drug encapsulation efficiency of all formulations was given in Table.2. It ranges from 53.12 to $97.73 \%$. The observed result reveals that the addition of cholesterol in niosomes increases its hydrodynamic diameter and entrapment efficiency. Literature reveals that cholesterol increases the chain order of liquid-state bilayers and decreases gel state bilayers. At higher concentration the gel state of cholesterol is transformed to a liquid-ordered phase. The rationale for the delayed drug release rate from the encapsulated formulation is an increase in cholesterol content of the bilayers in the formulation and also it gives an increase of the rigidity of the bilayers. The enhancement in the entrapment volume is due to the presence of charge that tends to increase the interlamellar distance between successive bilayers in multilamellar vesicle structure. ${ }^{13}$

\section{Characterization of Gel}

Colour, clarity, $\mathrm{pH}$, viscosity of the plain and niosomal gel lie down in the optimum $\mathrm{pH}$ range of skin. It avoids the occurrence of irritation upon the application to the skin. The drug release through the gel depends upon the viscosity also and all the prepared formulations were optimum in its viscosity and results were shown in Table 2 .

\section{Characterization of Transdermal Patch}

All the patches were evaluated for its weight uniformity, folding endurance, thickness, and percentage moisture content and the results given in Table 3. The weight 
uniformity of all formulations ranges from 44.1 to $53.36 \mathrm{mg}$ and the thickness of the patches varied from 0.257 to 0.295 $\mathrm{mm}$. The folding endurance was found to be $>150$ revealed that the prepared patches were having the capability to withstand the mechanical pressure along with good flexibility. \% moisture content of all patches ranges from 4.73 to 6.13 . The result shows that the lowest percent moisture absorption was found in Formulation TN4 when compared to other formulations. This is due to less aqueous permeability of ethyl cellulose polymer. The less moisture content in the formulations makes it to remain stable and from being a completely dried and brittle film. Additionally, microbial contamination and bulkiness of the patches can be protected by less moisture uptake. ${ }^{11}$

\section{Scanning Electron Microscopy}

The Particle Shape and surface characteristics of all formulations were examined by Scanning Electron Microscopy and figure 1 illustrated F3c. Noisome were found to be spherical in shape and formed good surfactant bilayered spheres.

\section{In-Vitro Release Studies on Topical Gel}

In general, the rate and time of drug release is predicted by drug release characterization. Based on sustained release performance in a formulation we can ensure the significance of polymer dissolution on drug release from matrices. ${ }^{4}$ Invitro release characteristics of all prepared formulation were studied and compared with standard. The results were illustrated in figures 2. \% drug release of niosomes was higher when compared to its standard. This result inconsistent with the previous results that the increasing the proportion of cholesterol: surfactant decreasing leakage of bilayer structure and thus released drug over $24 \mathrm{~h}$. The probable mechanism behind this is while increasing the proportion of cholesterol led to more rigid lipid bilayers as a barrier for drug release and decreased its leakage by improving the fluidity of the bilayer membrane and reducing its permeability, which led to lower drug release from the vesicles. ${ }^{16}$

\section{In-Vitro Release Studies on Transdermal Patch}

In-vitro release study on niosomal dispersion and niosomes, niosomal gel loaded transdermal patches was performed. The cumulative $\%$ drug release illustrated in Figure 2. Transdermal patch consists of PVP, Eudragit L 100, EC polymer and niosomal gel shown better release when compared to other formulations. This is inconsistent with the previous report by Shirsand SB et al., (2012). ${ }^{17}$

\section{Kinetic Model of In Vitro Drug Release Studies}

The in vitro release studies data of all formulations were fitted to various kinetic models like Zero order, First order, Higuchi Plot and Korsmeyer-Peppas plot to identify the drug release pattern 12 results shown in Table.3. The results indicate that all the formulations followed zero-order drug release with non-Fickian diffusion.

\section{CONCLUSIONS}

PC encapsulated vesicular carrier as transdermal patches is a promising drug delivery system to enhance the solubility of poorly soluble drugs. It also enhances the residence time of drug at absorption site. Hence this approach could be beneficial for the effective management of RA.

Financial or Other Competing Interests: None.

\section{REFERENCES}

[1] Aletaha D, Smolen JS. Diagnosis and management of rheumatoid arthritis: a review. JAMA 2018;320(13):1360-72.

[2] Sahu CR. Mechanisms involved in toxicity of liver caused by piroxicam in mice and protective effects of leaf extract of hibiscus rosa-sinensis L. Clin Med Insights Arthritis Musculoskelet Disord 2016;9:9-13.

[3] Bachhav AA, Jadhav AG, Pawar AY, et al. Development of proniosomal drug delivery with different type of penetration enhancers. International Journal of Pharm Tech Res 2018;11(1):57-73.

[4] Cherukuri S, Batchu UR, Mandava K, et al. Formulation and evaluation of transdermal drug delivery of topiramate. Int J Pharm Investig 2017;7(1):10-7.

[5] Patel KK, Kumar P, Thakkar HP. Formulation of niosomal gel for enhanced transdermal lopinavir delivery and its comparative evaluation with ethosomal gel. AAPS Pharm Sci Tech 2012;13(4):1502-10.

[6] EI-Ridy MS, Yehia SA, Mohsen AM. Formulation of niosomal gel for enhanced transdermal lornoxicam delivery: in-vitro and in-vivo evaluation. Curr Drug Deliv 2018;15(1):122-33.

[7] Ramesh K, Chandrashekar B, Khadgapathi P. Formulation and evaluation of poorly soluble etravirine by spray drying method. Int J Pharm Pharmsci 2015;7(4):98-103.

[8] Essa EA. Effect of formulation and processing variables on the particle size of sorbitan monopalmitate niosomes. Asian J Pharm 2010;4(4):227-33.

[9] Asthana GS, Asthana A, Singh D, et al. Etodolac containing topical niosomal gel: formulation development and evaluation. J Drug deliv 2016;2016:1-8.

[10] Dey SK, De PK, Sen T, et al. Formulation and in vitro evaluation of transdermal matrix patches of diclofenac sodium. J Pharm Res 2011;4(10):3593-6.

[11] Dahodwala ST, Sheikh A. Formulation and evaluation of transdermal film of promethazine hydrochloride. J Adv Pharm Edu Res 2013;3(4):559-65.

[12] Shefrin S, Sreelaxmi CS, Vijayan V, et al. Anti-epileptic drug loaded niosomal transdermal patch for enhanced skin permeation. Int J App Pharm 2019;11(2):31-43.

[13] Chandu VP, Arunachalam A, Jeganath A, et al. Niosomes: a novel drug delivery system. International Journal of Novel Trends in Pharmaceutical Sciences 2012;2(1):2531. 
[14] Sezgin-Bayindir Z, Antep MN, Yuksel N. Development and characterization of mixed niosomes for oral delivery using candesartan cilexetil as a model poorly watersoluble drug. AAPS PharmSci Tech 2015;16(1):108-17.

[15] Rehman M, Rasul A, Waqas MK, et al. Development and in vitro characterization of niosomal formulations of immunosuppressant model drug. Pak J Pharm Sci 2018;31(6 Suppl):2623-8.
[16] Ghanbarzadeh S, Khorrami A, Arami S. Nonionic surfactant-based vesicular system for transdermal drug delivery. Drug Deliv 2015;22(8):1071-7.

[17] Shirsand SB, Para MS, Nagendrakumar D, et al. Formulation and evaluation of ketoconazole niosomal gel drug delivery system. Int J Pharm Investig 2012;2(4):201-7. 